\title{
Minimal Cost Notice Board Using IoT
}

Selva Kumar VS ${ }^{1}$, Padmanaban R $^{2}$, Pradeep Kumaran R $^{2}$

${ }^{1}$ Associate Professor, Department of Electronics \& Communication Engineering, Rajalakshmi Engineering College, Chennai, Tamil Nadu, India

2Student, Department of Electronics \& Communication Engineering Rajalakshmi Engineering College Chennai, Tamil Nadu, India

\begin{abstract}
Article Info

Volume 8, Issue 2

Page Number : 415-421

Publication Issue

March-April-2021

Article History

Accepted : 01 April 2021

Published : 03 April 2021

As we know the purpose of noticeboards in schools and colleges had become unappealing among students. Also, the traditional way of sticking up the charts and paperwork was found to be dreary which is also why students are not actively involving in doing that. In this paper, we are proposing a model of online digital noticeboard using IOT which plays a vital role in reducing paper work, time and man power.

Here we use Node Miro-Controller Unit ESP8266 which has an in-built Wi-Fi module capable of sending/receiving data to/from internet. This microcontroller is connected with a LED dot matrix display which displays the data sent by the micro-controller.

We have also introduced some new concepts like collecting and displaying current time, date and day along with displaying the notice sent to the microcontroller through internet.

Keywords : Node MCU ESP8266, LED dot matrix MAX7219, smart noticeboard, Flutter, Firebase
\end{abstract}

\section{INTRODUCTION}

The problems with putting up paper notices on noticeboards are many in number: heavy wastage of paper, limitation in number of notices that can be put up at a time, recording and reuse of notices is rendered impossible, etc. Our study visualizes a smart screen hung outside every class-a smart system that regularly updates itself from a server, and effectively displays class-specific notices. The project is an IoT project in its true essence.
Node MCU plays a vital role in transmitting the information from mobile application to the smart display.

We have interfaced LED dot matrix display for long range and attractive visibility of the content. This smart display is cost effective and also looks attractive enough to get people's attention. Knowing time, date and current day is a vital part of every day. Most of the classes in schools and colleges lacks a clock. This 
system displays time, date, and day continuously in between displaying notices. The network time protocol helps us to feed current time and date in this instance. We use Google Cloud Realtime database to store and retrieve notices.

\section{Internet Of Things - Services and Components used}

Node MCU ESP8266: The micro controller unit that connects to the internet and fetches and displays data from the databases.

Network Time Protocol API: Current accurate time and date are provided by Network Time Protocol. After connecting to the internet, time and date are obtained from NTP servers using its API by the micro-controller.

Firebase Real-Time Database: It provides a database and a secure connection to access the data. The changes given by the user through mobile phone is instantly reflected on the notice board because of this Firebase Real-Time Database.

Firebase Authentication: This service verifies the user's credentials and gives appropriate access to the user to access the database.

\section{EXISTING SYSTEMS}

Ethernet module is used as a source for internet connectivity [1][6]. Here ethernet cables are laid along till the notice board and connected to the ethernet module. Raspberry $\mathrm{Pi}$ is used as a primary micro-controller [5][7][2] and large LCD monitors are used as display. Here images can be sent as a notice.

Some existing systems use a GSM modem, and combine it effectively with a basic LCD display. GSM modem with a SIM card is interfaced to the ports of the microcontroller with the help of AT commands. These systems make use of simpler micro-controllers like 8051, Atmel AT89C52, ATMEGA32 etc. A mobile phone is usually made use of in the client side to upload notices.[4]
Web applications are made in some papers using HTML CSS. This web application is used to change the contents of the noticeboard [5].

\section{SCOPE AND MOTIVATION}

In traditional method the notifications are displayed on the wooden noticeboard by writing the content on the paper which consumes space, prone to tampering and require manual labour to put and remove on time. Some important notices need to be displayed without any delay. So, there is a need for having smart digital notice board.

Government school students or students from rural schools tend to find schools boring or not interesting. A wireless display which shows time and notices will make them more interested to go to schools. If we speak about installing a device across all schools, then cost would be a major issue.

Our motive is to make schools very much interesting by introducing a smart device which is capable of making the class look futuristic within a minimal budget. Cost is our main objective.

The cost of this system would be,

Total Cost $=$ Cost of Micro-Controller + Cost of Display + Cost of power source

Total Cost $=150+200+50=₹ 400 /-$

This system provides less functionality than others but it is also much cost efficient than other available systems.

\section{METHODOLOGY}

\section{General Architecture}

We have used Firebase as a backend for the project. The Node MCU will constantly listen to the changes in the firebase real-time database and updates the LED Matrix display. Node MCU also fetches time, date and day from NTP servers and updates the LED 
Matrix display. The user's mobile phone will be authenticated using firebase authentication and listens to the changes in database. User's device has provision to change the contents of the database.

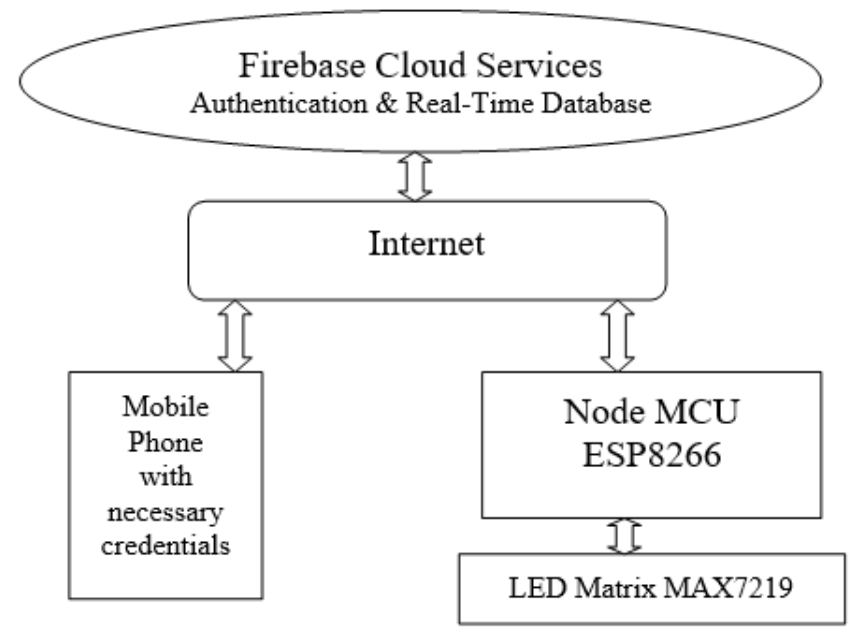

Fig 1.0 Architecture of the Minimal Cost Notice board system

\section{Notice Board Architecture}

We now explain the logic of working of our Node MCU system. For this we need Node MCU ESP8266 connected with LED Matrix MAX7219. We also need a known wi-fi connection nearby so that the device will connect to it. First the device connects to the known wi-fi connection thereby it connects to the internet. Once connected to the internet, it fetches time and date from the NTP server using APIs and displays it one by one on the LED matrix display. Using the predefined credentials present in the device, an access is given to the device to access the database. Now it gets the data from the firebase realtime database and displays it on the display in a scrolling manner. Now it again fetches time and date. This process is repeated.

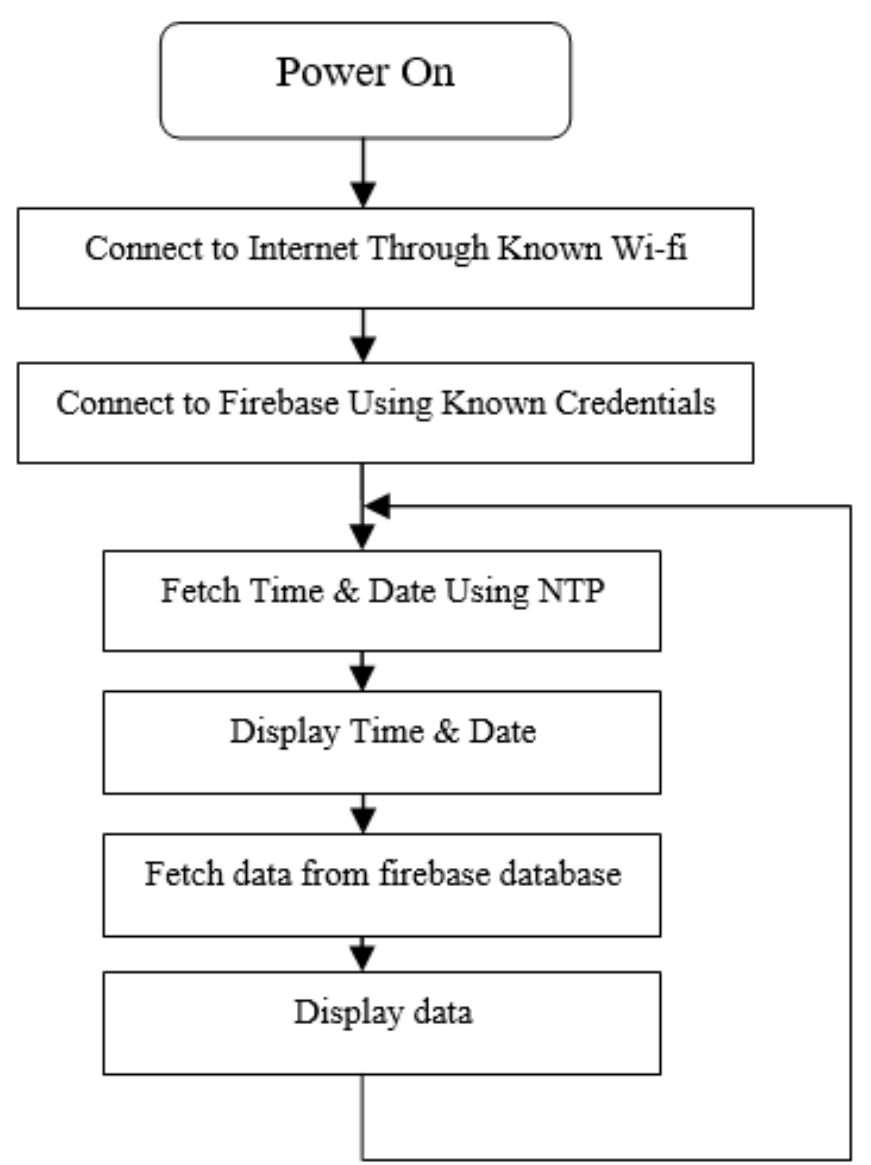

Fig 1.1 Flow chart for the notice board

\section{Mobile Application Architecture}

The mobile application starts with a login screen where user's credentials are entered. When login button is clicked the credentials are sent to firebase and firebase authentication verifies the credentials and provides access to the user to access the real-time database. (This process requires the mobile phone to be connected to the internet) Now the edit screen appears and we can edit the content to be displayed on the notice board. Once the edit button is clicked the new text is sent to the database which replaces the old one. This is also reflected on the device. 


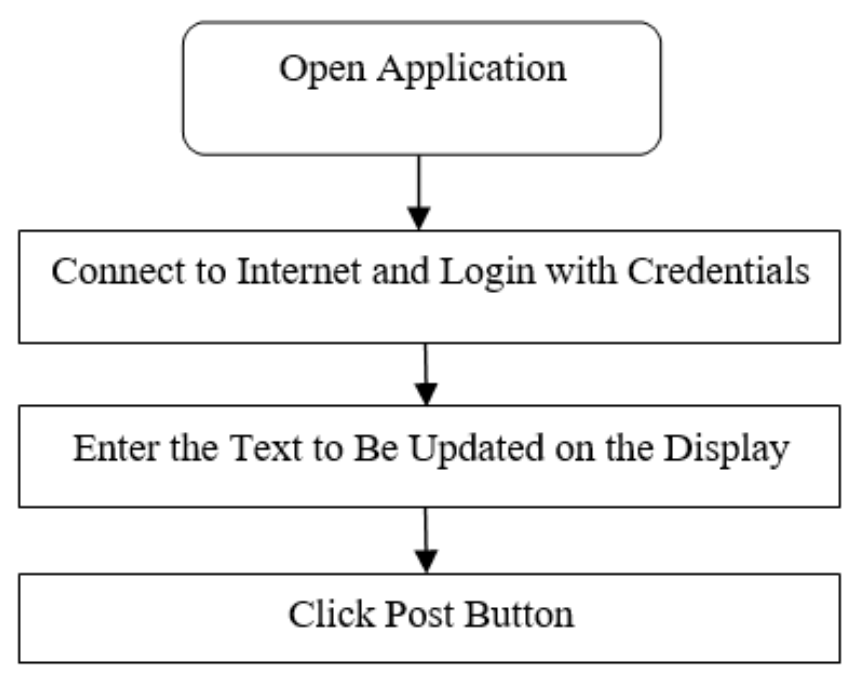

Fig 1.2 Flow chart for the mobile application

\section{Justification for Opted Methodology}

Firebase provides universal access to the notice board (although it requires internet on both mobile and noticeboard). Using Node MCU with LED Dot Matrix would be the most cost-effective way of implementing it. Mobile application made using flutter frame-work is more efficient than other app development ways as this app can be migrated to IOS or Windows with minimal changes. Even web version can be created from the mobile app within few steps.

\section{IMPLEMENTATION}

\section{Technology Stack}

This system uses efficient technology stack which provides results with minimal delay and high reliability. It also provides high number of functionalities at a low cost.

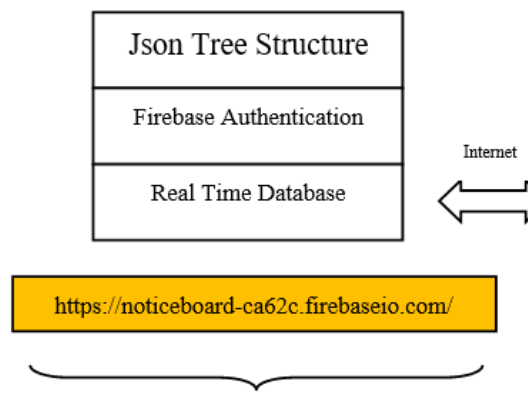

Server Side

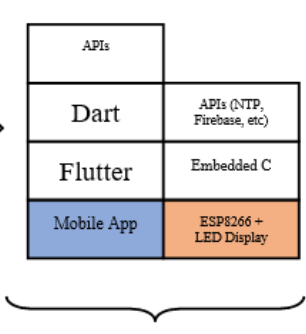

Client Side
Fig 2.0 Technology Stack for the noticeboard system
The server side is hosted on firebase. The whole backend process is handled by firebase. On the client side, flutter is used to make the mobile application and embedded $\mathrm{c}$ language is used to make the microcontroller program. Flutter is a framework which is written and used by the language dart. The various services used in this system has their own APIs which allows us to fetch data from them (Example: NTP, Firebase, etc).

\section{Dot Matrix Type Display}

Dot Matrix LED MAX7219 Array is used in this system. The two main advantages of using this type of display are,

\section{High Visibility}

2. Low Cost and Power Efficient

The colour scheme (black and red) and its intensity (of LEDs) makes sure that the display is visible from a long distance. Comparing to other display methods (like using LCD monitors), this method is highly power saving and cost saving.

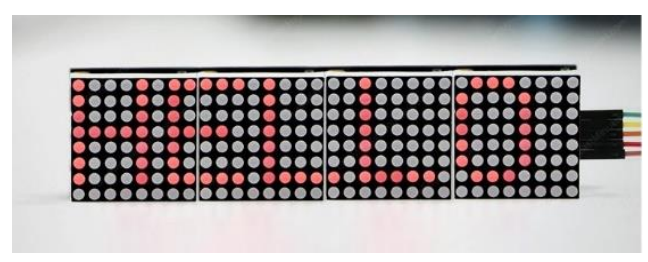

Fig 2.1 Image of our noticeboard displaying time

\section{Wifi Micro-controller}

This project uses Node MCU ESP8266 as its microcontroller. This kind of microcontrollers provide functionality to connect to the internet or a Local Area Network (LAN) and send/receive data accordingly. It is built such a way that its processing powers are limited thus reducing the cost of the device. Since it has networking capabilities it can be used in IoT related applications. 


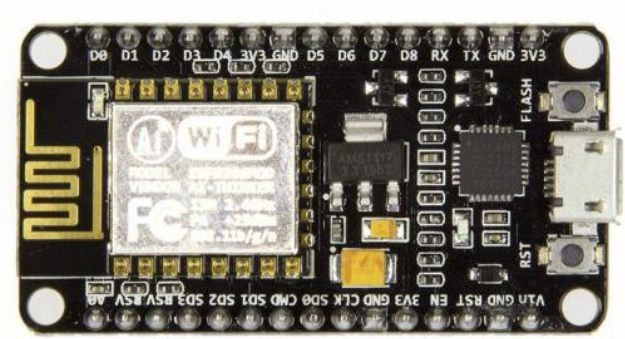

Fig 2.2 Image of Node-MCU ESP8266

\section{Flutter App}

The mobile application that is used in this project is made using flutter. Flutter is a framework that can be used to make applications. It is written in Dart language. The code for the app is also written in dart language. While making the app, important API keys and credentials related to firebase are saved into the application. This app consists of 2 screens, the login screen and the edit screen. The login screen insists users to enter the credentials (email ID and password). It then uses the saved keys to contact firebase authentication service and verifies the credentials. If the credentials are right, then the app shows its second screen which is the edit screen. Here you can see the text that is currently present on the noticeboard. You also have provision to edit the text. When you edit the text, the app uses the firebase keys and credentials to change the data present in the database. This whole process will not work if there is no internet connection available.

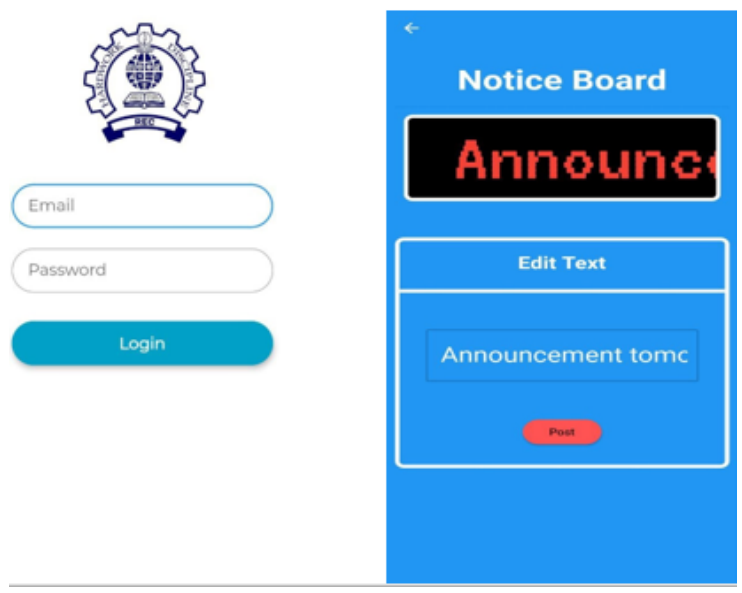

Fig 2.3 Screenshots from the mobile application

\section{Firebase Backend}

Firebase is used as a backend for the project. Firebase is a platform developed by google that provides cloud services at a minimal cost. This project uses firebase for 2 purposes,

\section{User Authentication \\ 2. Real Time Database}

Firebase authentication prevents any unauthorized person to access and change the contents of the noticeboard. It uses high level encryption and provides better security than any other backend services. Firebase Real-time database acts as a cloud storage of data where we can access it anytime and anywhere. Google is one of the biggest cloud service providers in the world. So, firebase can be used as backend service with high reliability.

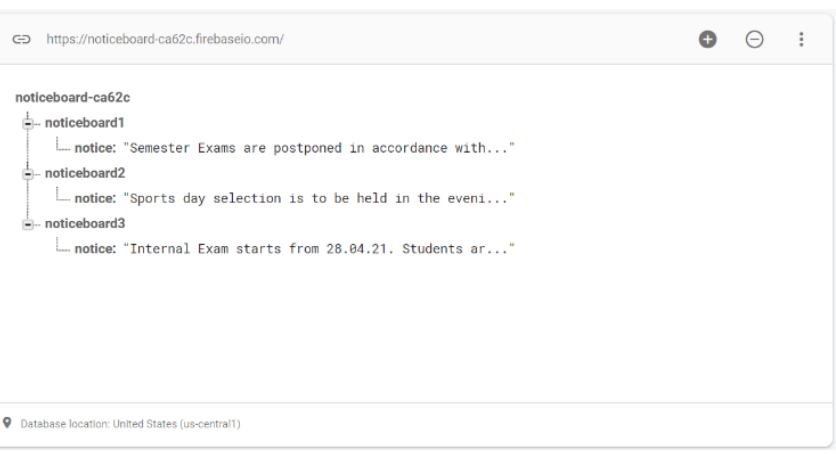

Fig 2.4 Image of firebase real-time database

The above image shows the structure of the firebase real time database that is used in this project. We can have any number of noticeboards connected to any number of users.

\section{APPLICATION OF LOW COST NOTICE BOARDS}

Cloud-based smart noticeboards will be a big part of smart homes and smart cities as it implies the concepts of IoT similar to smart homes and smart cities. Apart from that, these noticeboards can be placed anywhere like schools, colleges, shops, malls, etc. For example, when a shop is closed unexpectedly and he has to give a notice, he can type out his notice in the app and it will be displayed on the shop's notice board. It can be integrated with smart home 
and used at the door to give notice to people coming to our house.

\section{CONCLUSION}

The prototype of this cost-effective wireless noticeboard was efficiently designed. This prototype has facilities to be integrated with a display board thus making it truly mobile.

\section{Unit Testing}

This device is tested to last for more than 12 hours without any heating issues. The mobile application holds good without any crashes. Firebase backend works all time without any server issues.

\section{Future Work / Add-On Possibilities}

This is just a prototype of a wireless display board whose content can be changed using mobile phones. There is always room for changes. For instance, we can use buzzers to notify incoming notifications. We can also use speakers to speak out the notification text. Different sized dot matrix displays can be used based on locations and necessity.

\section{VII.ACKNOWLEDGMENT}

We would like to acknowledge the support provided by teaching and non-teaching staffs of department of Electronics \& Communication Engineering, Rajalakshmi Engineering College through required assistance during the project work. We also would like to thank our parent and classmates for their moral support and valuable suggestions in the project.

\section{REFERENCES}

[1]. Dinesh K, M Siva Ramakrishna, "IOT Based Digital Notice Board" published at International Journal of Engineering Research in Electronics and Communication Engineering (IJERECE) March 2017.
[2]. Divyashree M, Harinag Prasad S, Sandeep G T, Bhavya S N, Poornima S "IoT based webcontrolled notice board" published at International Research Journal of Engineering and Technology (IRJET) April 2017.

[3]. Piroj Pharaskhanewala, Maninder Kaur Rehal, Adarsh Wagh, Prof. Kalpana Malpe, "Wireless Notice-Board Using Node MCU", published at International Journal on Future Revolution in Computer Science \& Communication Engineering. January 2018.

[4]. Pooja Pawar, Suvarna Langade, Mohini Bandgar, "A Paper on IOT Based Digital Notice Board using Arduino ATMega 328" published at International Research Journal of Engineering and Technology (IRJET) March 2019.

[5]. Shivam Maheshwari, Vatsalya Singhal, Vijay Ravi, "Cost-effective and self-regulating digital Noticeboard systems" published at "3rd IEEE International Conference on Computational Systems and Information Technology for Sustainable Solutions" March 2018

[6]. Satish D. Jadhav, Yogita Mistry, "IOT BASED ELECTRONIC NOTICE BOARD" published at Technical Research Organisation India, November 2017.

[7]. Vishnu K M, Lalkrishna M D, Mohammed Farshan V T, Anu P M, Nivya Mariya Francis Anooja.B, "IOT Based Digital Notice Board" published at International Journal of Advanced Information Science \& Technology. April 2018

[8]. Baby.M., Divya.G., Harini.P\&Eleena Slesser.Y. "SMS Based Wireless E-Notice Board" published at IEEE Computer Society. 2012

[9]. Darshankumar C. Dalwadi, Ninad Trivedi , AmitKasundra, "WIRELESS NOTICE BOARD", published at IEEEComputer Society, 13-14 May 2011

[10]. Victor Chukwudi Osamor, Olatobi S. Aloba and Ifeoma P. Osamor , "From Wooden to Digital Notice Board (DNB): Design and Implementation for University 
Administration", IEEE Computer Society, 1010502-6868 IJECS-IJENS (C) April 2010

[11]. Nicolas Villar, Kristof Van Laerhoven, Hans Gellersen, "Physical notice board with digital logic and display", IEEE Computer Society, Embedded Interactive Systems Group Computing Department, Lancaster University, UK, 2012

[12]. Adil Bashir, Sama Qazi, Shoeib Amin Banday, Liyaqat Nazir, Bisma Shah, "Dual Tone Multi Frequency (DTMF) based Smart Notice Board System" IEEE Computer Society, E\&C Dept. National Institute of Technology, Srinagar, J\&K India E\&C, Islamic University of Science and Technology, J\&K India,2013, Volume 3, Issue 11

[13]. Jagan Mohan Reddy.N., Venkareshwarlu.G., Cbit, "Wireless Electronic Display Board Using Gsm Technology", IEEE Computer Society, Issn:2320-2084, Hyderabad-2013, Volume-1, Issue-10

[14]. Pudumai Nayagi, R.Seethalakshmi, "Design and Implementation of Digital Notice Board Using Power Line Communication", IEEE Computer Society, M.Tech Embedded Systems, School of Computing, SASTRA Universty, Thanjavur, Tamil Nadu, India,No 2 Apr-May 2013, ISSN : 0975-4024 Vol 5

[15]. Ajinkya Gaikwad, Tej Kapadia, Manan Lakhani \& Deepak Karia, "Zigbee Based Wireless Electronic Notice Board with Multi Point Recievers", IEEE Computer Society, Department of Electronics and ISBN: 978-15386-6078-2 (C) 2018 IEEE 246 Telecommunication Sardar Patel Institute of Technology, Mumbai 400058, India, 2013, ISSN (Print) : 2319 - 2526, Volume-2, Issue-3

[16]. Foram Kamdar1, Anubbhav Malhotra and Pritish Mahadik, "Display message on Notice Board using GSM", IEEE Computer Society, Department of Electronics and Telecommunications, Mumbai University,
S.P.I.T, Andheri West, Mumbai, INDIA., 2013, Volume 3, Number 7 (2013)

[17]. Gowtham.R, Kavipriya.K, Kesavaraj.G , Natheena.A , Mr. Maragatharaj.S. "Multiuser Short Message Service Based Wireless Electronic Notice Board", IEEE Computer Society, Knowledge Institute Of Technology, Salem, India UG Students, Department Of Electronics and Communication Engineering, ,Volume 2 Issue 4 April, 2013

\section{Cite this article as :}

Selva Kumar V S, Pradeep Kumaran R, Padmanaban R, "Minimal Cost Notice Board Using IoT", International Journal of Scientific Research in Science and Technology (IJSRST), Online ISSN : 2395-602X, Print ISSN : 2395-6011, Volume 8 Issue 2, pp. 415-421, March-April 2021. Available at doi : https://doi.org/10.32628/IJSRST218265 Journal URL : https://ijsrst.com/IJSRST218265 